\title{
Introduction
}

\section{Adult spinal deformity: pathophysiology and corrective measures}

Adam S. Kanter, M.D. ${ }^{1}$ Christopher I. Shaffrey, M.D. ${ }^{2}$ Praveen Mummaneni, M.D., ${ }^{3}$ Michael Y. Wang, M.D., ${ }^{4}$ AND JUAN S. URIBE, M.D. ${ }^{5}$

\begin{abstract}
${ }^{1}$ Department of Neurosurgery, University of Pittsburgh, Pittsburgh, Pennsylvania; '2Department of Neurological Surgery, University of Virginia Health System, Charlottesville, Virginia ${ }^{3}$ Department of Neurological Surgery, University of California, San Francisco, California; ${ }^{4}$ Department of Neurosurgery, University of Miami; and ${ }^{5}$ Department of Neurosurgery, University of South Florida, Tampa, Florida
\end{abstract}

This issue of Neurosurgical Focus is devoted to adult spinal deformity-its pathophysiology and corrective measures.

In the United States and around the world, the population is living longer and staying active. Individuals now experience degenerative scoliosis associated symptoms in their 6th and 7th decades of life, and they are increasingly disinclined to live with symptoms of back pain, radiculopathy, and postural instability that limit their mobility and active lifestyles.

Treatment of this patient population is fraught with potential short-term morbidity. Major spinal reconstruction procedures are associated with perioperative complications including iatrogenic anemia, neurological deficits, prolonged hospitalization, and the prospect of pseudarthrosis and hardware failure from contributory osteoporosis.

Contemporary surgical techniques have mitigated some of these issues. In particular, minimally invasive deformity surgery has been shown to decrease blood loss, infection rate, and length of hospitalization, but significant spino-pelvic deformity correction remains a challenge when less invasive techniques are employed. Ad- ditionally, not all patients with deformity are appropriate candidates for minimally invasive spinal surgery; this can be due to anatomical constraints, limitations of the procedures themselves, or pathology severity, such as patients with severe kyphoscoliosis who often require open osteotomies to optimize spinal realignment.

The current edition of Neurosurgical Focus provides information on the latest options to treat patients with adult spinal deformity. The articles highlight several key parameters including radiographic outcomes following circumferential minimally invasive spinal correction techniques and clinical outcomes, as well as the cost effectiveness of these procedures in comparison to traditional open procedures. Complications following threecolumn reconstruction are evaluated, including variables such as patient characteristics, pathology, and surgical approach. Unilateral versus bilateral iliac screws are appraised. Algorithms are proposed for cervical deformity and treatment strategies to aid in decision making for minimally invasive surgery regarding lumbar deformities. And finally, an evolutionary perspective detailing the origins of lumbar lordosis and the mechanics and biology of lumbar degeneration is uniquely described.

Spinal deformity surgery continues to evolve, and the articles in the current issue will aid in its evolution. We thank the many authors involved and encourage continued pragmatic examination of this complex pathological entity. (http://thejns.org/doi/abs/10.3171/2014.3.FOCUS14112)

\section{Disclosure}

Dr. Kanter receives royalties from Biomet. Dr. Uribe is a consultant for NuVasive. Dr. Mummaneni receives royalties from DePuy Spine, QMP Publishing, and Thieme Publishers; he receives honoraria from Globus; and he owns stock in Spinicity. Dr. Wang is a consultant for and patent holder in DePuy Spine. Dr. Shaffrey is a consultant for Biomet, Globus, Medtronic, NuVasive, and Stryker, and he is a patent holder in and receives royalties from Biomet and Medtronic. 